\title{
High-Performance Embedded Morphological Wavelet Coding
}

\author{
F. Lazzaroni, R. Leonardi and A. Signoroni* \\ Dept. of Electronics for Automation, Signal and Communications Laboratory \\ University of Brescia, via Branze, 38, Brescia, I25123, Italy \\ Ph*:+39 030 3715432, Email*: signoron@ing.unibs.it, Web: scl.ing.unibs.it
}

\begin{abstract}
Morphological analysis can be applied in wavelet domain to analyze and represent the position of significant coefficients. New operators have to be introduced which are able to exploit both the multiresolution and the filter bank peculiarities of the subband representation of visual information. In this paper an efficient morphological wavelet coder is proposed. The clustering trend of significant coefficients is captured by a new kind of multiresolution binary dilation operator. The layered and adaptive nature of this subband dilation makes it possible for the coding technique to produce an embedded bit-stream with a modest computational cost and stateof-the-art Rate-Distortion performance. Morphological wavelet coding appears promising because the localized analysis of wavelet coefficient clusters is adequate to capture intrinsic patterns of the source which can have substantial benefits for perceptual or even object-based reconstruction quality concerns. Here we test the performance of our algorithm and compare the effects of different wavelet filters. We obtain state of the art coding performance and good perceptual results both for $2 D$ and $3 D$ images, with a new technique that seems to be well suited for further developments.
\end{abstract}

\section{INTRODUCTION}

Among the best image compression schemes, wavelet based ones currently provide for high Rate-Distortion (R-D) performance with a low computational complexity. The zerotree-based approach is widespread since it reaches high performance by exploiting the intersubband dependency among insignificant wavelet coefficients. This is mainly achieved by reorganizing such coefficients in scale-space trees. The zerotree idea was first introduced by Shapiro's EZW [1] and then reformulated with the SPIHT algorithm by Said and Pearlman [2]. Besides the zerotree concept, other techniques which exploit statistical dependencies in the wavelet domain have been recently proposed. For example the "Embedded Block Coding with Optimized Truncation" (EBCOT) [3] algorithm, adopted in the JPEG2000 standard [4], combines layered block coding, R-D optimization and context-based arithmetic coding in an efficient and highly scalable way.

The zerotree idea can also be reformulated in a dual way, where one builds significance maps by dealing directly with significant coefficients. To do this a morphological

This research has been partially supported by the IST programme of the EU under projects IST-2001-32795 SCHEMA. processing has been used and also justified by the statistical evidence of energy clustering in the wavelet subbands [5]. The "Significance-Linked Connected Component Analysis" (SLCCA) algorithm [6], has shown compression performance similar to the best known zerotreebased algorithms. However, SLCCA does not provide a progressive fidelity bit-stream.

In this work, we propose a new embedded wavelet coding strategy, based on a new multiresolution dilation operator (EMDC) which strengthens the "morphocodec" idea and outperforms both morphologic and conventional low complexity state-of-the-art codecs. As we will see, a combined and priority layered action of significant coefficient dilations and an adaptive scanning procedure around the cluster boundaries are able to capture the complex nature of subband clusters in a bit-plane quantization framework and to produce a highly R-D optimized progressive bit-stream. Finally we perform an evaluation of some different wavelet filters with reference to our coder, and compare the performance of EMDC with respect to the most popular wavelet-based coders, both on 2D and 3D data.

\section{AN EMBEDDED MORPHOLOGICAL APPROACH TO WAVELET CODING}

Recognizing and modeling the statistical properties of wavelet-transformed natural images is a crucial task in the design of a high-performance wavelet-based coder, and previous works such as $[1,2,3,7]$ have been successful in doing so. In fact, the significant-insignificant dichotomy and the exploitation of high-order (structured) statistical relationships in some space-frequency domain are implemented both in the quantization and in the entropy coding stages. However, zerotree based algorithms do not completely exploit an important statistical property of wavelet-transformed natural images: the clustering trend of most significant coefficients (in the sense that their module is larger than a certain threshold). We show an example of this phenomena in Fig.1, where we can observe some big clusters in correspondence to textured regions (as the Lena's hair) and objects boundaries (as the Lena's hat, shoulder and eyes). A possible approach to exploit this behavior has been introduced with the morphological conditioned dilation coding $[5,6]$.

We want to show how the morphological approach may be efficiently extended within an embedded framework using the already detected significant coefficients as a 


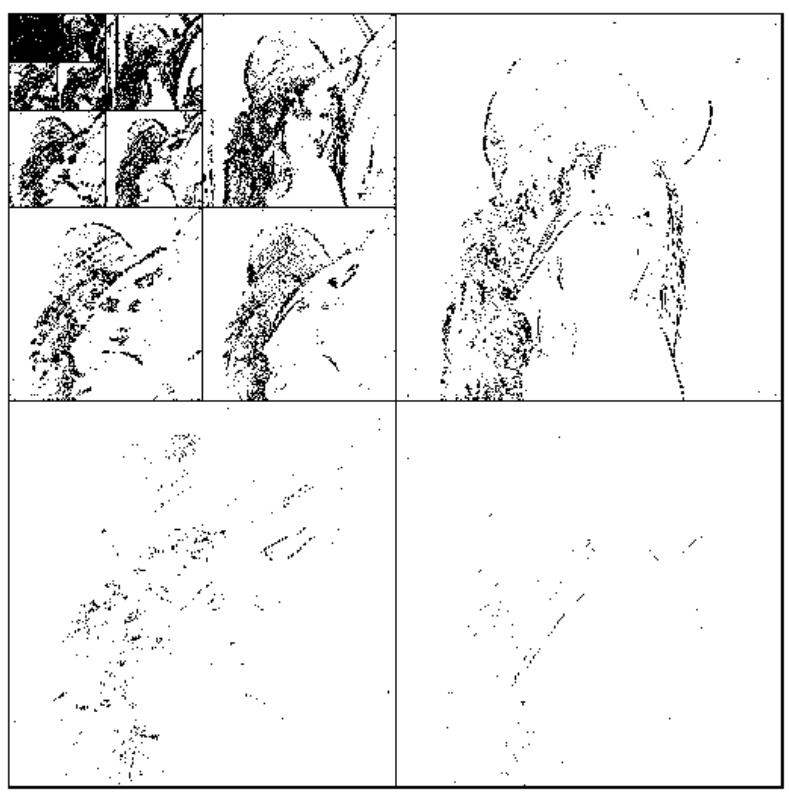

Figure 1: Significance-map of a four-level decomposition of the image Lena.

base for the search of new significant ones. This approach is justified by the observation that clusters tend to grow, in the scale-space wavelet domain, when crossing successive bit-planes.

Here we introduce a new dilation operator which lets improve the coding performance without affecting the computational efficiency, and makes it possible to overcome some open questions, emerging from previous works: a) Servetto et al. [5] give an excellent justification of the morphological approach to better represent significance maps, but use it in an intra-band mode only, letting the entropy coder exploit some inter-band statistical dependencies; b) Chai et al. [6] define a bit-saving inter-band linking mechanism among subband clusters. However, the coder architecture does not produce an embedded bit-stream, and good R-D performance are also due to a pre-processing stage which eliminates all isolated significant coefficients (without considering their magnitude), whose positions are expensive to code. Moreover, in [6], the assessment accuracy of the cluster boundaries is viewed as a static tradeoff between structuring element extent and related bit cost.

The main innovations introduced here with respect to the previous approaches are the subdivision of the morphological dilation in different layers ordered in base of their expected R-D performance, and an original approach to the analysis of the regions around the cluster boundaries.

\section{THE EMBEDDED MORPHOLOGICAL DILATION CODING}

We call our algorithm "Embedded Morphological Dilation Coding": EMDC. As in the popular zerotree coders, our progressive EMDC algorithm is composed of two iterative stages: a sorting pass that predicts and identifies significant coefficients (i.e. larger than a given threshold) and codes their position, and a refinement pass that adds to the precision of the previously marked significant coefficients.

The dilation operator is applied on a layered structure: (a) an intra-band morphological dilation and (b) two steps of inter-band expansion, which allow to explore the already identified clusters; (c) a boundary morphological dilation which analyzes the coefficients on the cluster boundaries; and (d) an explicit position coding which describes isolated significant coefficients and identifies new clusters ("seed layer"). These five stages analyze regions with decreasing expected frequencies of identification of significant coefficients, as shown in Fig.2. Moreover, the different dilation stages do not intervene in a sequential order. In fact, the intra-band morphological dilation is executed every time a new significant coefficient is found. This way, for each bit-plane, the R-D performance of the coded information are optimized, as we briefly show in the following.

If the frequency of identification of significant coefficients in a particular region is equal to $f_{i}$, the expected coding rate $R_{i}$ in this region (without considering contextual information) can be approximated to the zero-order entropy:

$$
H_{i}=-f_{i} \log _{2}\left(f_{i}\right)-\left(1-f_{i}\right) \log _{2}\left(1-f_{i}\right)
$$

The distortion reduction rate is instead proportional to the frequency $f_{i}$ :

$$
\Delta D_{i}=f_{i} E\left[\Delta D_{s i g}\right]
$$

where $E\left[\Delta D_{\text {sig }}\right]$ is the expected distortion reduction when a significant coefficient is found in the considered bit-plane.

The average distortion decrease for coded bit can be therefore approximated, for each dilation layer $i$, as:

$$
\delta_{i}=\frac{\Delta D_{i}}{H_{i}}
$$

This set of values $\delta_{i}$ is plotted, for the example of Fig.2, in Fig. 3 on the reference curve:

$$
\delta(f)=-\frac{f E\left[\Delta D_{\text {sig }}\right]}{f \log _{2}(f)+(1-f) \log _{2}(1-f)} .
$$

with $f \in(0,1)$. This demonstrates that the layered structure of the dilation brings good R-D behavior into the embedded bit-stream.

Another important feature of our dilation strategy is the capability to detect multiresolution clusters (by the stage (b)) and to work on an extended connectivity basis in order to go beyond false cluster boundaries. In fact, typically on the boundaries of a big cluster, there are a few scattered significant coefficients but it is very difficult to forecast the dimension of the area interested to this phenomenon. Therefore, the extent of the searching area around a cluster may not be fixed a-priori. Indeed, the hereinafter described algorithm searches around clusters of significant coefficients at increasing distance as long as new significant coefficients can be found: the width of the scanned area is adaptively selected on the 


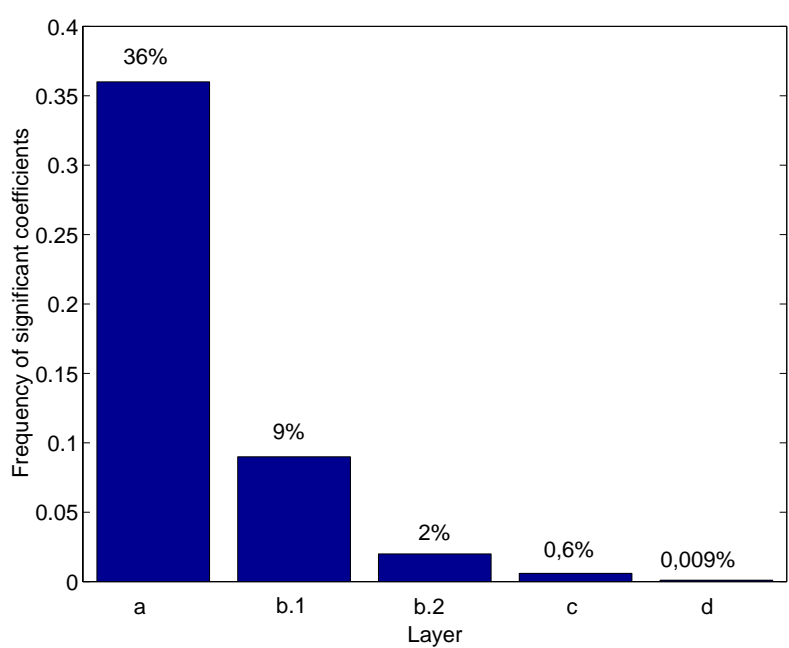

Figure 2: A typical example of frequencies of significant coefficients occurrence for the 5 coding layers of a bitplane.

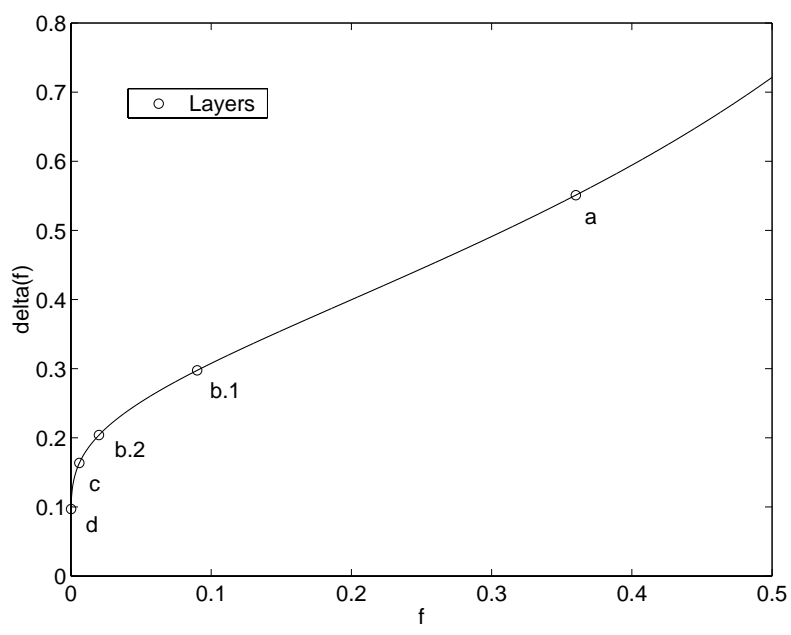

Figure 3: Average distortion reduction per coded bit in function of the frequency of significant coefficients, eq.(3),(4). The operating points corresponding to the layer frequencies $f_{i}$ of Fig. 2 are emphasized.

basis of the occurrence of newly significant coefficients. This mechanism is implemented through the iterative dilation of insignificant-marked coefficients (stage (c)). All this actually entails a new kind of morphological operator, and allows to overcome the static tradeoff mentioned at the end of the previous section.

In the rest of the paper we shall use this notation: $c=c(x, y)$ for the generic wavelet coefficient; $S C L_{l, i}$, $I C L_{l, i}$ for the significant and insignificant coefficient lists of the $(l, i)$ subband respectively; $\mathrm{AC}(d)$ to represent the arithmetic coding of $d ; S_{n}(c)$ to identify the significance magnitude test by the threshold $2^{n}$, "true" for significant, "false" for insignificant; $V_{\eta}(c)$ to define the vicinity of $c$ in a same subband and with the $\eta$ neighborhood system; $s(c)$ to identify the sons of $c$ in the tree subband structures.

The coding process works as follows:
Initialization: $M=\max (c) ; n=\left\lfloor\log _{2}(M)\right\rfloor$, output $n$; set all the $S C L_{l, i}$ and $I C L_{l, i}$ as empty lists.

1. Significance-map morphological-coding (sorting pass):

(a) Intra-band morphological dilation: for each subband $(l, i)$ do:

- $\forall c \in S C L_{l, i}$ consider $\widetilde{c} \in V_{\eta}(c)$ that have not been already scanned during this sorting pass.

- if $S_{n}(\widetilde{c}): \mathrm{AC}(1), \mathrm{AC}(\operatorname{sign}(\widetilde{c}))$, add $\widetilde{c}$ to the end of $S C L_{l, i}$; otherwise: $\mathrm{AC}(0)$, add $\widetilde{c}$ to the end of $I C L_{l, i}$.

(b) Inter-band expansion:

- step 1: for each subband $(l, i)$ do:

- $\forall c$ in the father subband's, which has been marked as significant in a previous sorting pass, $S C L_{l-1, i}$ the four sons $s(c)$ are considered, except those already scanned during this sorting pass.

- if $S_{n}(\widetilde{c})$, with $\widetilde{c} \in s(c): \mathrm{AC}(1)$, $\mathrm{AC}(\operatorname{sign}(\widetilde{c}))$, add $\widetilde{c}$ to the end of $S C L_{l, i}$ and immediately perform 1(a); otherwise: $\mathrm{AC}(0)$, add $\widetilde{c}$ to the end of $I C L_{l, i}$.

- step 2: identical to step 1 but for the coefficient which has been marked as significant during the current sorting pass;

(c) Boundary morphological dilation: for each subband $(l, i)$ do:

- $\forall c \in I C L_{l, i}$ except those included during this step 1(c), consider $\widetilde{c} \in V_{\eta}(c)$ except those already scanned during this sorting pass.

- if $S_{n}(\widetilde{c}): \mathrm{AC}(1), \mathrm{AC}(\operatorname{sign}(\widetilde{c}))$, add $\widetilde{c}$ to the end of $S C L_{l, i}$ and immediately perform 1(a); otherwise: $\mathrm{AC}(0)$, add $\widetilde{c}$ to the end of $I C L_{l, i}$.

- Repeat 1(c) only on the new entries of $I C L_{l, i}$ until two successive scans do not lead to new significant coefficients.

(d) Explicit position coding: for each subband $(l, i)$ do:

- The position of the remaining significant coefficients $\widetilde{c}$ is explicitly sent; add $\widetilde{c}$ to $S C L_{l, i}$ and immediately perform 1(a).

- A special character is sent when all significant coefficients in the subband $(l, i)$ have been marked.

2. Bit-plane refinement: for each entry in all $S C L$ 's, except those included in the last sorting pass, AC(the $n$th most significant bit);

3. Bit-plane update: empty all $I C L_{l, i}$, decrement $\mathrm{n}$ by 1 and repeat from step 1 . 


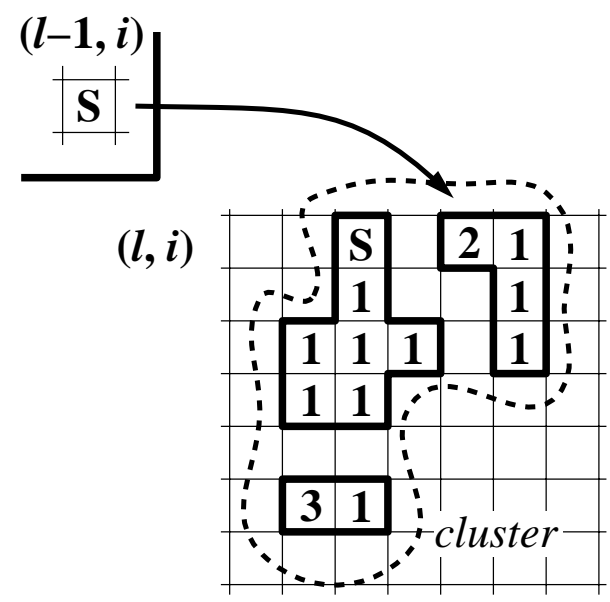

Figure 4: Example of layered dilation. $\mathrm{S}$ are the seeds for the dilations; 1, 2 and 3 correspond to the significant coefficients found during intra-band morphological dilation, inter-band expansion and additional morphological dilation respectively. The dashed boundary idealizes an unique cluster.

This algorithm can be easily adapted to compression of volumetric data, extending the $\eta$ neighborhood system and the subband structure to the three dimensions as exposed in a precedent version of the algorithm [8]. Note that the first significant coefficient is always coded by an explicit position coding, but this operation is then performed very rarely in the rest of the coding. The algorithm can be stopped in any moment at the desired rate or distortion.

\section{ENTROPY CODING}

In order to increase the coding efficiency, the bitstream produced by the progressive quantization is entropy coded using a context-based adaptative arithmetic coder, based on [9]. First, we partition the coding bitstream in three: significance-map coding bits, signs bits and refinement bits.

The significance-map coding bits are entropy compressed exploiting the relationship between neighboring coefficients both in spatial and in resolution domain. In fact, as we have already observed, it is more probable that a coefficient is significant if it is near other significant coefficients and if its father in the tree subband structure is significant. Besides, we propose to make use in some case (see also [10]) of three significance values for the father coefficients, distinguishing not only between significant and insignificant, but also between previouslysignificant (marked as significant in a previous sorting pass) and newly-significant (marked in the current sorting pass).

Thus, for the significance-map coding we identify 10 different contexts (both for 2D and 3D data):

- 7 contexts for intra-band morphological dilation:

- 1 context for the first level subbands;

- 6 contexts for the other subbands as a combination of the three significance value of the father (previously-significant, newly-significant or insignificant) and the following binary condition: we verify the presence of at least one adjacent significant coefficient towards the direction of the low-pass subband filter (the $\mathrm{HH}$ subband coefficients are considered without such significant coefficients); with this condition we partially allow for the different directional behavior of a separable subband representation;

- 2 contexts for inter-band expansion, depending on the significance value of the father (previouslysignificant or newly-significant);

- 1 context for additional morphological dilation: in this step all the analyzed coefficients are far from the already significant-marked coefficients, and so we do not identify here sub-contexts.

Explicit position coding bits are not entropy compressed. The entropy coding is performed also on refinement bits, as described in [10], and on sign bits [3].

\section{EXPERIMENTAL RESULTS}

\subsection{Evaluation of the performance of different filters}

We evaluate the performance of our coder EMDC using three different filters: the 9/7-tap filters [11], the 10/18-tap filters [12] and the more recent 22/14-tap filters [13]. We choose the three 8-bit $512 \times 512$ gray-scale images Lena, Barbara and Goldhill. As shown in Tab.1, Tab.2 and Tab.3, the main differences are obtained on the compression of Barbara image, where the 22/14-tap filters outperform both the 10/18-tap and more remarkably the 9/7-tap filters. Besides, also on the images Lena and Goldhill 22/14 and 10/18 filters appear superior to the 9/7 one, and this can be distinctly observed also in terms of visual (perceptual) quality in Fig.5. We ascribe this behavior to the good significance cluster representation property of EMDC which allows to effectively exploit the higher number of vanishing moment of the 10/18-tap and 22/14-tap wavelet basis.

Table 1: PSNR performance for the "Lena" image of the EMDC coder with different filters.

\begin{tabular}{|c|c|c|c|}
\hline Rate (bpp) & $9 / 7$ & $10 / 18$ & $22 / 14$ \\
\hline \hline 0.25 & 34.50 & 34.38 & 34.60 \\
\hline 0.50 & 37.57 & 37.48 & 37.66 \\
\hline 1.00 & 40.50 & 40.42 & 40.52 \\
\hline
\end{tabular}

Table 2: PSNR performance for the "Barbara" image of the EMDC coder with different filters.

\begin{tabular}{|c|c|c|c|}
\hline Rate (bpp) & $9 / 7$ & $10 / 18$ & $22 / 14$ \\
\hline \hline 0.25 & 28.40 & 28.21 & 28.60 \\
\hline 0.50 & 32.15 & 32.30 & 32.61 \\
\hline 1.00 & 37.38 & 37.82 & 37.84 \\
\hline
\end{tabular}


Table 3: PSNR performance for the "Goldhill" image of the EMDC coder with different filters.

\begin{tabular}{|c|c|c|c|}
\hline Rate (bpp) & $9 / 7$ & $10 / 18$ & $22 / 14$ \\
\hline \hline 0.25 & 30.74 & 30.77 & 30.76 \\
\hline 0.50 & 33.55 & 33.50 & 33.57 \\
\hline 1.00 & 36.94 & 37.00 & 37.00 \\
\hline
\end{tabular}

\subsection{Comparison with other morphocodecs}

In Tab.4 we compare our Embedded Morphological Dilation Coding performance with the best results obtained by the MRWD[5] and SLCCA[6] morphocodecs; both are not embedded ${ }^{1}$ ( $9 / 7$ filters are used everywhere). $0.48 \mathrm{~dB}$ and $0.26 \mathrm{~dB}$ are the average PSNR improvements of EMDC with respect to MRWD and SLCCA respectively.

Table 4: PSNR results on 2D images, measured in $\mathrm{dB}$.

\begin{tabular}{|l|c|c|c|c|}
\hline Image & Rate (bpp) & $\begin{array}{c}\text { MRWD } \\
{[5]}\end{array}$ & $\begin{array}{c}\text { SLCCA } \\
{[6]}\end{array}$ & EMDC \\
\hline \hline \multirow{3}{*}{ Lena } & 0.25 & 34.12 & 34.28 & 34.50 \\
& 0.50 & 37.18 & 37.35 & 37.57 \\
& 1.00 & 40.33 & 40.47 & 40.50 \\
\hline \multirow{3}{*}{ Barbara } & 0.25 & 27.86 & 28.18 & 28.40 \\
& 0.50 & 31.44 & 31.89 & 32.15 \\
& 1.00 & 36.24 & 36.69 & 37.38 \\
\hline \multirow{3}{*}{ Goldhill } & 0.25 & 30.53 & 30.60 & 30.74 \\
& 0.50 & 33.15 & 33.26 & 33.55 \\
& 1.00 & 36.56 & 36.66 & 36.94 \\
\hline
\end{tabular}

\subsection{Comparison with popular wavelet codecs}

For 2D application we tested the EMDC algorithm on the image Lena, Barbara and Goldhill. For wavelet transform we used five-level decomposition with 22/14-tap filters [13]. We compared our coder with respect to the embedded coder SPIHT [2], and the JPEG2000 standard ${ }^{2}$ with the "single-rate" setting in order to obtain the best PSNR performance. However in this case JPEG2000 lose its scalability properties. As shown in Tab.5, Tab.6 and Tab.7, our algorithm shows the best performance with the following average PSNR gains: $0.65 \mathrm{~dB}$ over SPIHT and $0.35 \mathrm{~dB}$ over JPEG2000. A visual comparison is presented in Fig. 6 where different progressive coders are compared at the same rate of $0.125 \mathrm{bpp}$.

Table 5: PSNR performance for the "Lena" image.

\begin{tabular}{|c|c|c|c|}
\hline Rate (bpp) & SPIHT & JPEG2000 & EMDC \\
\hline \hline 0.25 & 34.11 & 34.14 & 34.60 \\
\hline 0.50 & 37.21 & 37.30 & 37.66 \\
\hline 1.00 & 40.41 & 40.40 & 40.52 \\
\hline
\end{tabular}

\subsection{EMDC volume coding}

For volumetric applications we tested the EMDC algorithm on the medical $256 \times 256 \times 128$ test volume

\footnotetext{
${ }^{1}$ There exists an embedded version of the MRWD codec, but with performance reducing by some tenths of $\mathrm{dB}$.

${ }^{2}$ We used the JPEG2000 codec software Kakadu available at http://www.kakadusoftware.com/ .
}

Table 6: PSNR performance for the "Barbara" image.

\begin{tabular}{|c|c|c|c|}
\hline Rate (bpp) & SPIHT & JPEG2000 & EMDC \\
\hline \hline 0.25 & 27.58 & 28.40 & 28.60 \\
\hline 0.50 & 31.39 & 32.22 & 32.61 \\
\hline 1.00 & 36.41 & 37.16 & 37.84 \\
\hline
\end{tabular}

Table 7: PSNR performance for the "Goldhill" image.

\begin{tabular}{|c|c|c|c|}
\hline Rate (bpp) & SPIHT & JPEG2000 & EMDC \\
\hline \hline 0.25 & 30.56 & 30.58 & 30.76 \\
\hline 0.50 & 33.13 & 33.25 & 33.57 \\
\hline 1.00 & 36.55 & 36.54 & 37.00 \\
\hline
\end{tabular}

CT_SKULL $[14,15]$ and compared our coding results with respect to others state-of-art coders $[14,15,10]$. We used for wavelet transform the 22/14-tap filters [13]. Our results are sensibly superior to those shown by other coders both in terms of mean PSNR on the whole volume, of worst slice PSNR and in terms of mean slice PSNR fluctuations among near slices [16]. The results are summarized in Tab.8.

\section{CONClusions AND PERSPECTIVES}

In this paper a new embedded morphological dilation coder (EMDC), has been proposed. This algorithm is based on a new kind of morphological dilation of significant coefficients that exploit inter-band and intra-band statistical dependencies among significant coefficients, their tendency to define clusters, and it is able to learn the morphologic nature of cluster boundaries. Moreover, the layered structure of the dilation guarantees a highly optimized R-D embedding and a fine structured bit-stream which allow a very efficient adaptive entropy coding. The EMDC algorithm shows performance often sensibly superior to the state-of-the-art wavelet based image coders. Besides, the EMDC morphocodec allows to directly inspect the morphological properties of the significant coefficient clusters: this places the algorithm in touch with perceptual coding principles and strategies. Some research perspectives can be expected in the field of image, volume and video coding, where perceptual (e.g. visually lossless coding) or semantic (e.g. region or object of interest selective coding) information may benefit from a connected cluster analysis integrated within the coding strategy.

Table 8: Mean PSNR (a), worst slice PSNR (b) and mean PSNR oscillation (c) on the 128 slice volume CT_SKULL.

\begin{tabular}{|c|c|c|c|c|c|}
\hline $\begin{array}{c}\text { Rate } \\
\text { (bpp) }\end{array}$ & & $\begin{array}{c}\text { EZW } \\
\text { 3D [14] }\end{array}$ & $\begin{array}{c}\text { SPIHT } \\
\text { 3D [15] }\end{array}$ & $\begin{array}{c}\text { I-SPIHT } \\
\text { 3D [10] }\end{array}$ & $\begin{array}{c}\text { EMDC-3D } \\
\text { 22/14-tap }\end{array}$ \\
\hline \hline \multirow{3}{*}{0.1} & $\mathrm{a}$ & $\simeq 32.5$ & 33.99 & 34.07 & 35.50 \\
& $\mathrm{~b}$ & 29.8 & 29.2 & 30.98 & 32.50 \\
& $\mathrm{c}$ & $\simeq 2.5$ & $\simeq 6.0$ & 2.28 & 2.32 \\
\hline \multirow{3}{*}{0.5} & $\mathrm{a}$ & $\simeq 42$ & 42.89 & 43.67 & 44.78 \\
& $\mathrm{~b}$ & 39.5 & 37.5 & 41.64 & 42.93 \\
& $\mathrm{c}$ & $\simeq 2.5$ & $\simeq 4.0$ & 2.39 & 2.05 \\
\hline
\end{tabular}




\section{REFERENCES}

[1] J. M. Shapiro, "Embedded image coding using zerotrees of wavelet coefficients," IEEE Trans. on Acoustics, Speech and Signal Processing, vol. 41, no. 12, pp. 3445-3462, Dec. 1993.

[2] A. Said and W. A. Pearlman, "A new fast and efficient image codec based on set partitioning in hierarchical trees," IEEE Trans. Circuits Syst. Video Technol., vol. 6, pp. 243-250, June 1996.

[3] D. Taubman, "High performance scalable image compression with EBCOT," IEEE Trans. Image Processing, vol. 9, pp. 1158-1170, July 2000.

[4] D. Taubman, E. Ordentlich, M. Weinberger, and G. Seroussi, "Embedded block coding in JPEG 2000," Sig. Proc.: Image Comm., vol. 17, pp. 4972, Jan. 2002.

[5] S. D. Servetto, K. Ramchandran, and M. T. Orchard, "Image coding based on a morphological representation of wavelet data," IEEE Trans. Image Processing, vol. 8, pp. 1161-1174, Sept. 1999.

[6] B. B. Chai, J. Vass, and X. Zhuang, "Significancelinked connected component analysis for wavelet image coding," IEEE Trans. Image Processing, vol. 8, pp. 774-784, June 1999.

[7] S.-T. Hsiang and J. W. Woods, "Embedded image coding using zeroblocksof subband/wavelet coefficients and context modeling," in MPEG-4 Workshop and Exhibition at ISCAS 2000, Geneva, Switzerland, May 2000.

[8] F. Lazzaroni, A. Signoroni, and R. Leonardi, "Embedded morphological dilation coding for 2D and 3D images," in VCIP 2002, San José, California, Jan. 2002, vol. SPIE(4671), pp. 923-934.

[9] A. Moffat, R. M. Neal, and I. H. Witten, "Arithmetic coding revisited," ACM Trans. Information Systems, vol. 16, pp. 256-294, July 1998.

[10] A. Signoroni, M. Arrigoni, F. Lazzaroni, and R. Leonardi, "Improving SPIHT-based compression of volumetric medical data," in PCS 2001, Seoul, Korea, Apr. 2001, pp. 187-190.

[11] M. Antonini, M. Barlaud, P. Mathieu, and I. Daubechies, "Image coding using wavelet transform," IEEE Trans. Image Processing, vol. 1, pp. 205-220, Apr. 1992.

[12] M. J. Tsai, J. D. Villasenor, and F. Chen, "Stackrun image-coding," IEEE Trans. on Circuits and Systems for Video Tech., vol. 6, no. 5, pp. 519-521, Oct. 1996.

[13] D. Wei, H-T Pai, and A. C. Bovik, "Antisymmetric biorthogonal coiflets for image coding," in ICIP' 98 , Oct. 1998, vol. 2, pp. 282-286.
[14] A. Bilgin, G. Zweig, and M. W. Marcellin, "Three-dimensional image compression using integer wavelet transforms," Applied Optics: Information Processing, Special Issue on Information Theory in Optoelectronic Systems, vol. 39, no. 3, pp. 1799-1814, Apr. 2000.

[15] Z. Xiong, X. Wu, D. Y. Yun, and W. A. Pearlman, "Progressive coding of medical volumetric data using three-dimensional integer wavelet packet transform," in VCIP'99, San José, California, Jan. 1999, vol. SPIE(3653), pp. 327-335.

[16] A. Signoroni and R. Leonardi, "Modeling and reduction of PSNR fluctuations in 3D wavelet coding," in ICIP 2001, Thessaloniki, Greece, Oct. 2001, p. WP07. 


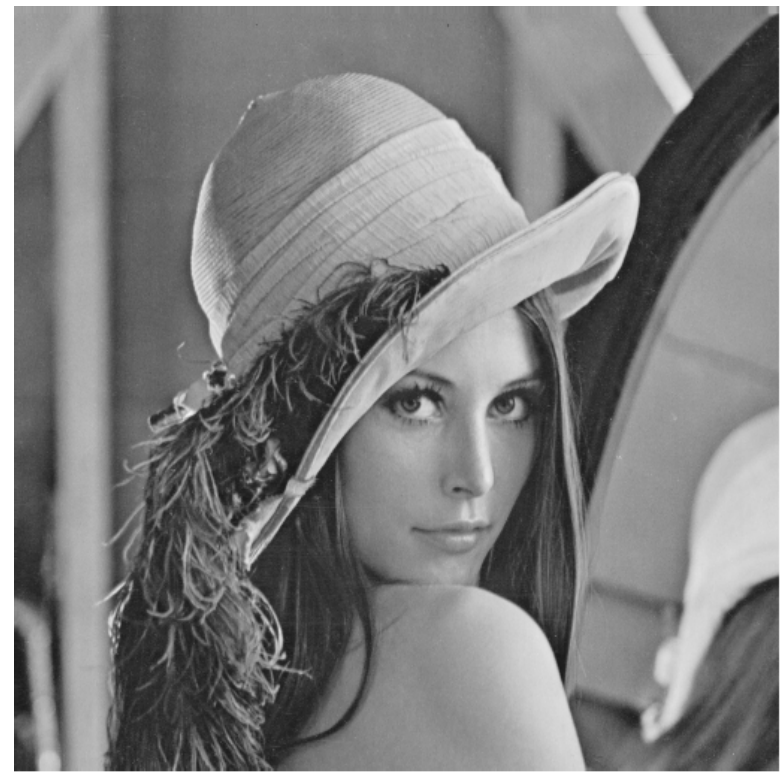

(a)

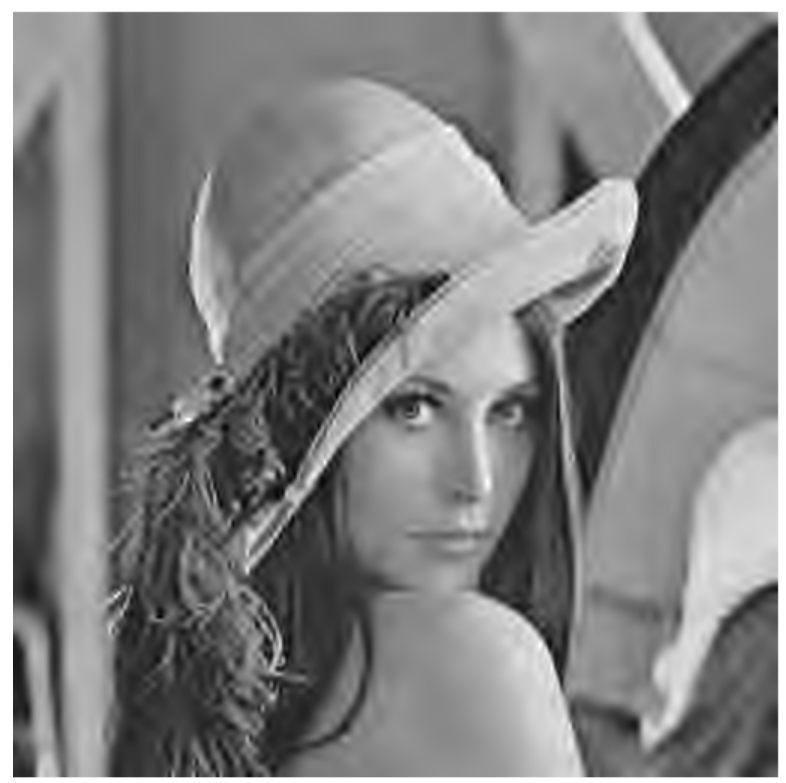

(c)

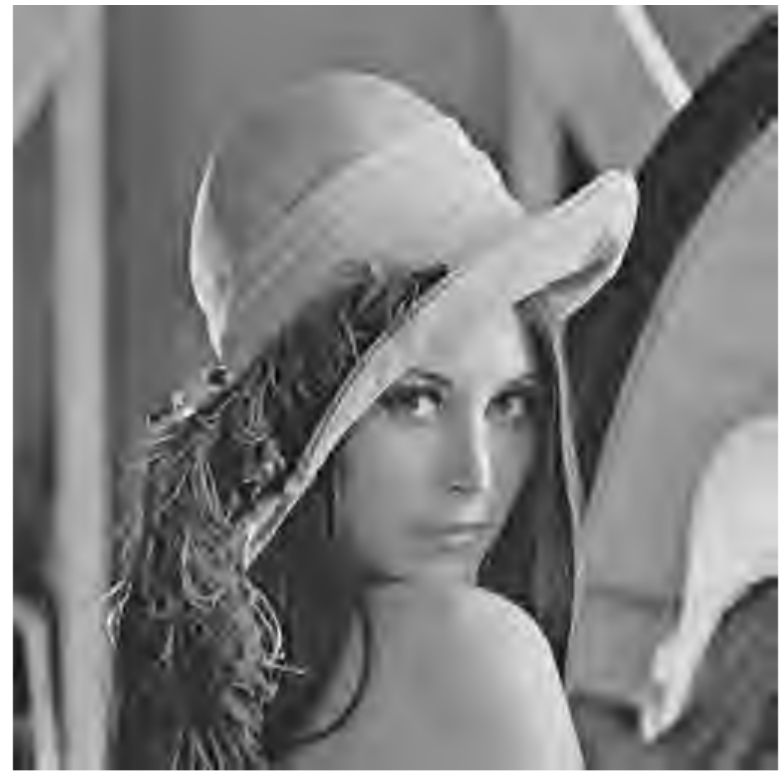

(b)

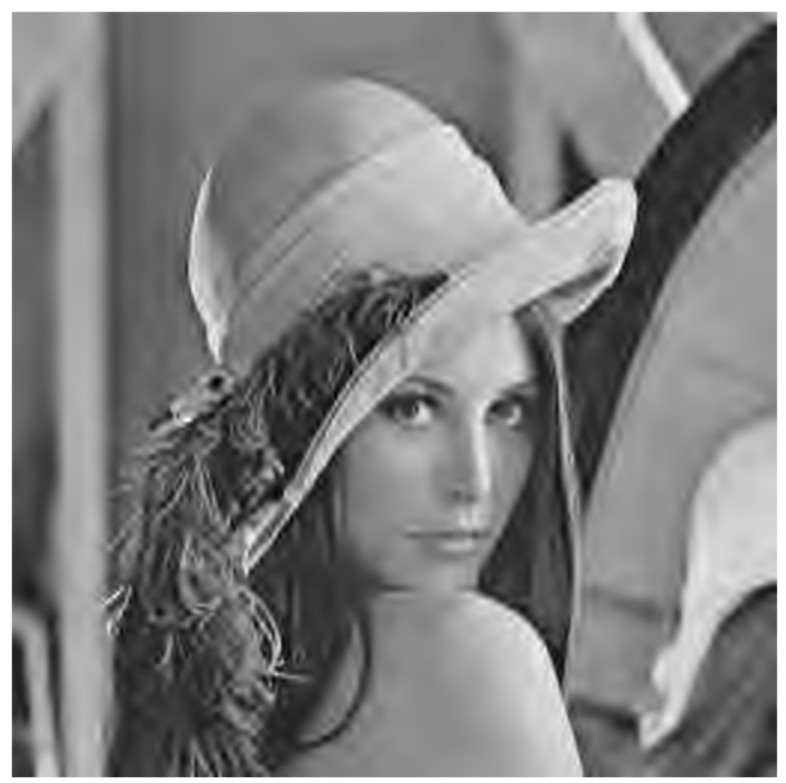

(d)

Figure 5: Coding results for image Lena at $0.0625 \mathrm{bpp}$. (a) Original image. (b) Compressed image with 9/7-tap filter. (c) Compressed image with 10/18-tap filter. (d) Compressed image with 22/14-tap filter. 


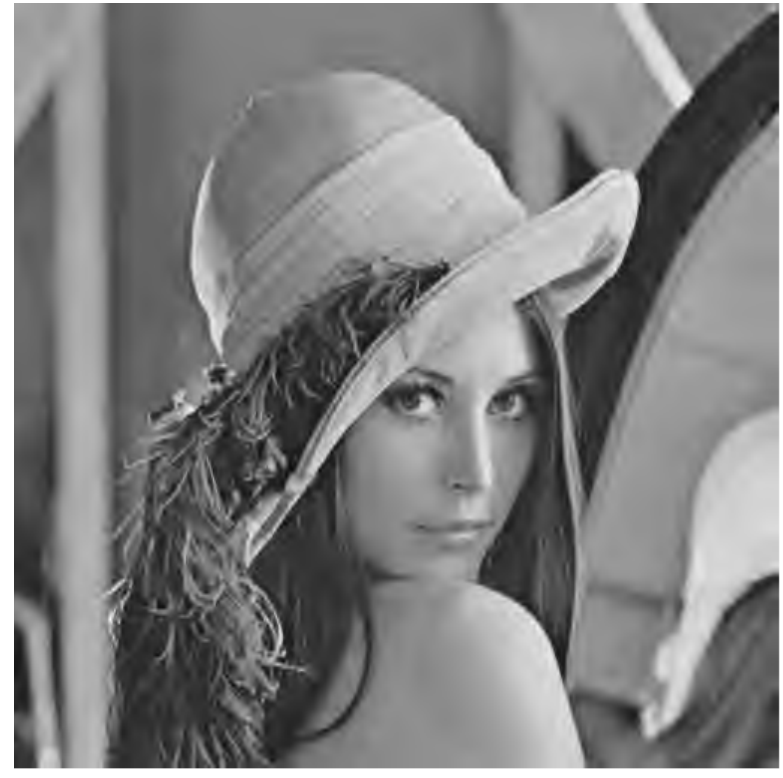

(a)

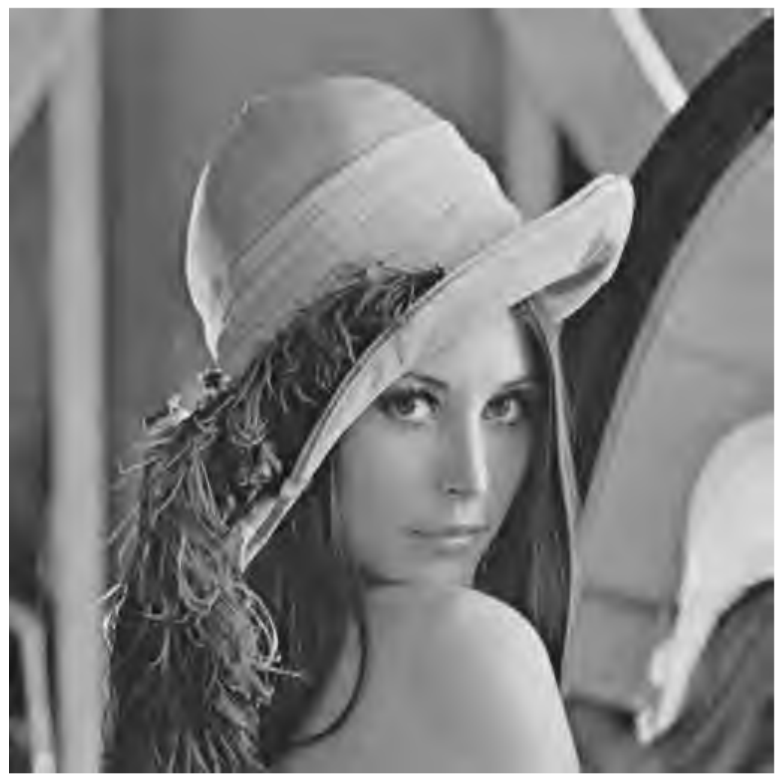

(c)

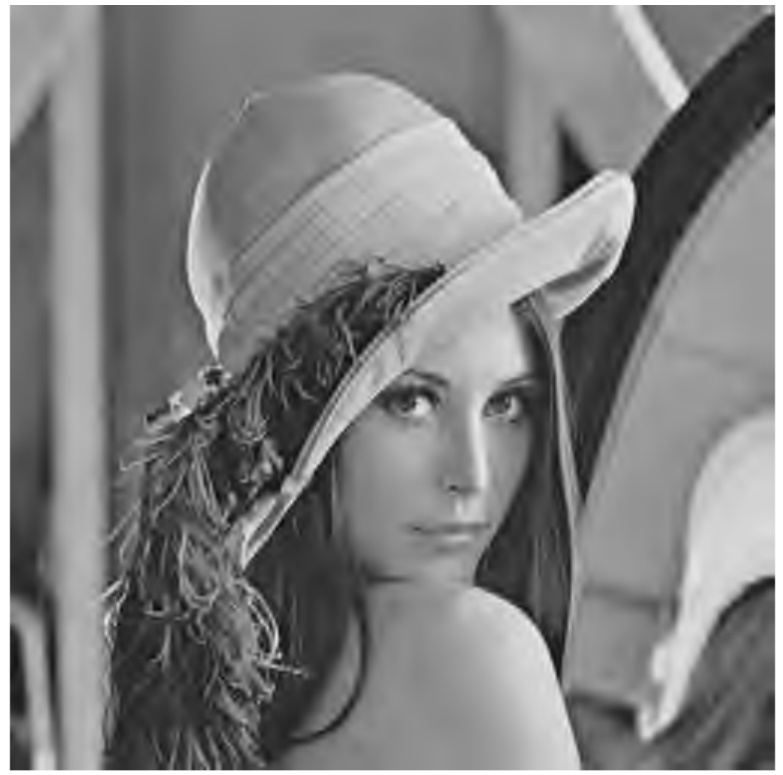

(b)

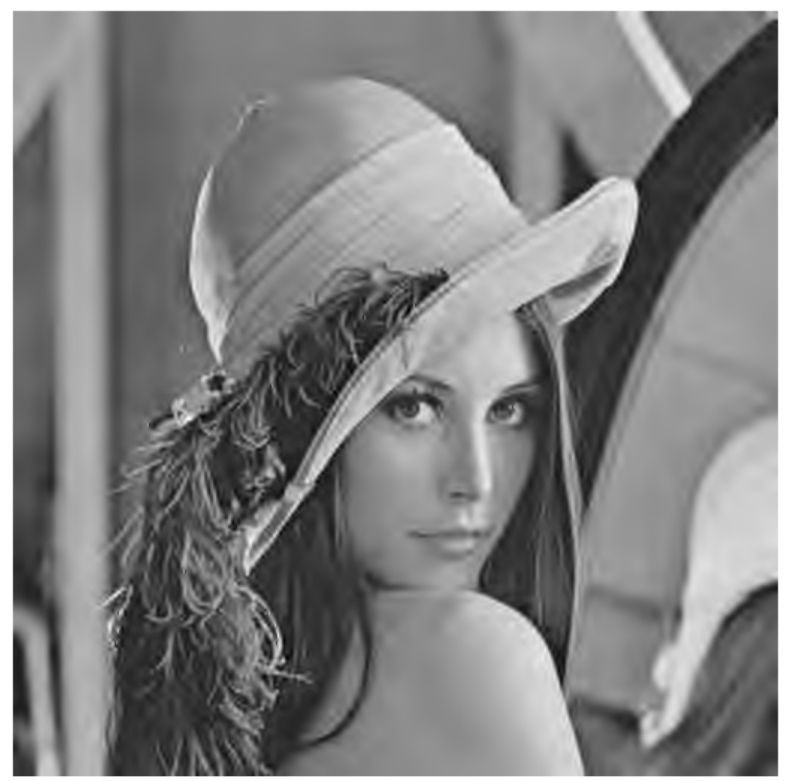

(d)

Figure 6: Coding results for image Lena at 0.125 bpp: (a) SPIHT (PSNR=31.10 dB); (b) "progressive" JPEG2000 with 50 quality layers (30.82 dB); (c) EMDC with 9/7-tap filters (31.46 dB); (d) EMDC with 22/14-tap filters (31.54 dB). 